\title{
Dirubidium tricadmium tetrakis(sulfate) pentahydrate
}

\section{Diptikanta Swain and}

\section{T. N. Guru Row*}

Solid State and Structural Chemistry Unit, Indian Institute of Science, Bangalore 560012 ,

Karnataka, India

Correspondence e-mail:

ssctng@sscu.iisc.ernet.in

\section{Key indicators}

Single-crystal X-ray study

$T=90 \mathrm{~K}$

Mean $\sigma(\mathrm{S}-\mathrm{O})=0.003 \AA$

$R$ factor $=0.022$

$w R$ factor $=0.059$

Data-to-parameter ratio $=11.0$

For details of how these key indicators were automatically derived from the article, see http://journals.iucr.org/e.
The title compound, $\mathrm{Rb}_{2} \mathrm{Cd}_{3}\left(\mathrm{SO}_{4}\right)_{4} \cdot 5 \mathrm{H}_{2} \mathrm{O}$, arose as an unexpected product during the attempted synthesis of an $\mathrm{Rb}_{2} \mathrm{Cd}_{2}\left(\mathrm{SO}_{4}\right)_{3}$ potassium cadmium sulfate langbeinite, It has two layers, layer $A$ containing Cd octahedra bridged by sulfate groups and layer $B$ containing edge-shared Cd octahedra, with $\mathrm{Rb}$ atoms occupying interstial positions. The layers are connected by way of $\mathrm{Cd}-\mathrm{O}-\mathrm{S}$ links.

\section{Comment}

The system $\mathrm{Rb}_{2} \mathrm{SO}_{4}-\mathrm{CdSO}_{4}-\mathrm{H}_{2} \mathrm{O}$ was selected in an attempt to synthesize the langbeinite-type phase $\mathrm{Rb}_{2} \mathrm{Cd}_{2}\left(\mathrm{SO}_{4}\right)_{3}$ by a slow evaporation method. Instead, the title compound, (I) (Fig. 1), a hydrated double salt, arose at $313 \mathrm{~K}$.

The crystal structure of (I) is isomorphous with $\mathrm{K}_{2} \mathrm{Mn}_{3}\left(\mathrm{SO}_{4}\right)_{4} \cdot 5 \mathrm{H}_{2} \mathrm{O}$ (Hidalgon et al., 1996). The Cd atoms are octahedrally coordinated by $\mathrm{O}$ atoms of either sulfate groups or water molecules (Table 1 ).

Among the five water molecules, $\mathrm{O} 18 \mathrm{~W}$ bonds to two $\mathrm{Cd}$ atoms $\left[\mathrm{Cd} 1-\mathrm{O} 18 W-\mathrm{Cd} 2=114.72(12)^{\circ}\right]$. The four other water molecules are singly coordinated to $\mathrm{Cd}$ atoms. The crystal packing (Fig. 2) results in pseudo-layers parallel to the $b c$ plane. Two types of layers, namely layer $A$ formed by $\mathrm{Cd}$ octhedra bridged by sulfate groups and layer $B$ containing edge-sharing $\mathrm{Cd}$ octahedra, occur. The pseudo-layers, which are connected by way of $\mathrm{Cd}-\mathrm{O}-\mathrm{S}$ bonds, repeat in a $\ldots B A B B A B \ldots$ fashion along the $a$ axis, with the $\mathrm{Rb}$ cations in interstitial positions. A network of $\mathrm{O}-\mathrm{H} \cdots \mathrm{O}$ bonds (Table 2) helps to consolidate the crystal packing.

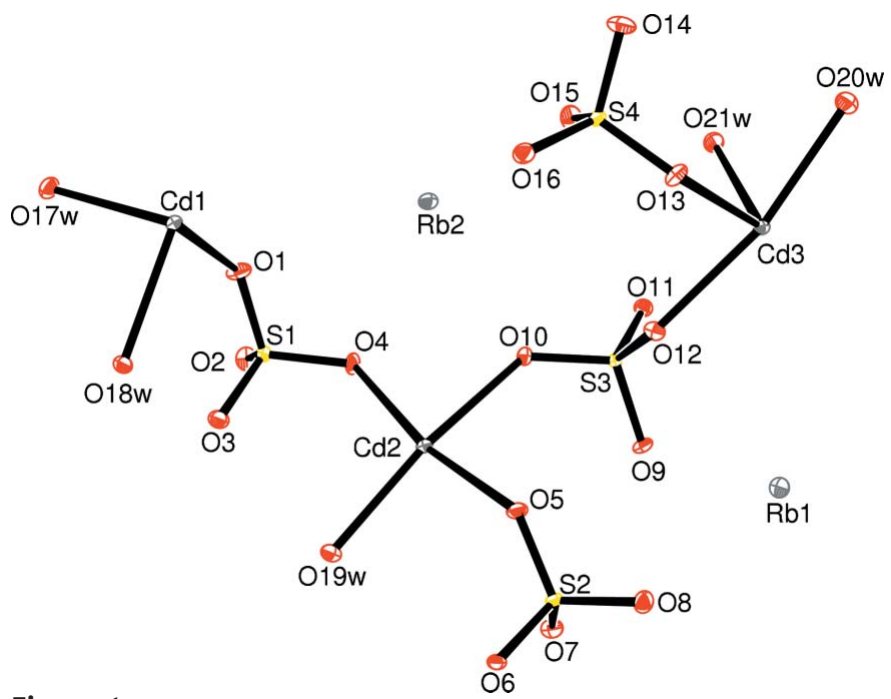

Figure 1

View of the asymmetric unit of (I), showing $50 \%$ displacement ellipsoids. $\mathrm{H}$ atoms have been omitted for clarity. 


\section{Experimental}

Colourless plates of (I) were synthesized by slow evaporation at $313 \mathrm{~K}$ of an aqueous solution containing equimolar amounts of $\mathrm{Rb}_{2} \mathrm{SO}_{4}$ and $\mathrm{CdSO}_{4}$. The temperature was maintained by a thermostat to control the evaporation rate.

\section{Crystal data}

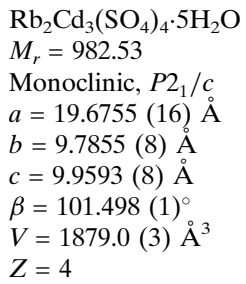

\section{Data collection}

$\begin{array}{ll}\text { Bruker SMART CCD } & 3434 \text { independent reflections } \\ \quad \text { diffractometer } & 3296 \text { reflections with } I>2 \sigma(I) \\ \varphi \text { and } \omega \text { scans } & R_{\text {int }}=0.031 \\ \text { Absorption correction: multi-scan } & \theta_{\max }=25.4^{\circ} \\ \quad(S A D A B S ; \text { Sheldrick, 1996) } & h=-23 \rightarrow 23 \\ T_{\min }=0.189, T_{\max }=0.755 & k=-11 \rightarrow 11 \\ 13435 \text { measured reflections } & l=-11 \rightarrow 11\end{array}$

\section{Refinement}

Refinement on $F^{2}$

$R\left[F^{2}>2 \sigma\left(F^{2}\right)\right]=0.022$

$w R\left(F^{2}\right)=0.059$

$S=1.10$

3434 reflections

311 parameters

$\mathrm{H}$-atom parameters constrained

\author{
$D_{x}=3.473 \mathrm{Mg} \mathrm{m}^{-3}$ \\ Mo $K \alpha$ radiation \\ Cell parameters from 890 \\ reflections \\ $\theta=0.9-28.7^{\circ}$ \\ $\mu=9.06 \mathrm{~mm}^{-1}$ \\ $T=90(2) \mathrm{K}$ \\ Plate, colourless \\ $0.29 \times 0.16 \times 0.03 \mathrm{~mm}$
}

3434 independent reflections

$l=-11 \rightarrow 11$

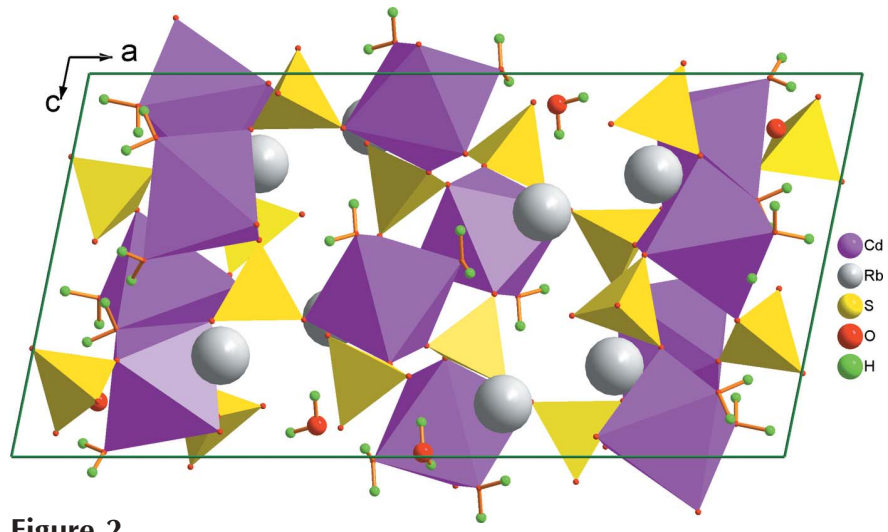

Figure 2

Packing diagram of (I).

Table 2

Hydrogen-bond geometry $\left(\AA{ }^{\circ}\right)$.

\begin{tabular}{lllll}
\hline$D-\mathrm{H} \cdots A$ & $D-\mathrm{H}$ & $\mathrm{H} \cdots A$ & $D \cdots A$ & $D-\mathrm{H} \cdots A$ \\
\hline $\mathrm{O} 17 W-\mathrm{H} 17 B \cdots \mathrm{O} 3^{\text {viii }}$ & 0.82 & 1.95 & $2.755(4)$ & 166 \\
$\mathrm{O} 18 W-\mathrm{H} 18 A \cdots \mathrm{O} 2^{\text {vii }}$ & 0.82 & 1.82 & $2.627(4)$ & 168 \\
$\mathrm{O} 18 W-\mathrm{H} 18 B \cdots \mathrm{O} 3$ & 0.82 & 1.79 & $2.599(4)$ & 169 \\
$\mathrm{O} 19 W-\mathrm{H} 19 A \cdots \mathrm{O} 4^{\text {vi }}$ & 0.82 & 2.02 & $2.807(4)$ & 162 \\
$\mathrm{O} 19 W-\mathrm{H} 19 B \cdots \mathrm{O} 17 W^{\text {v }}$ & 0.82 & 2.39 & $3.019(4)$ & 134 \\
$\mathrm{O} 20 W-\mathrm{H} 20 A \cdots \mathrm{O} 21 W^{\text {ix }}$ & 0.82 & 2.36 & $3.123(4)$ & 154 \\
$\mathrm{O} 20 W-\mathrm{H} 20 B \cdots \mathrm{O} 13^{\text {iv }}$ & 0.82 & 2.38 & $2.966(4)$ & 129 \\
$\mathrm{O} 20 W-\mathrm{H} 20 B \cdots \mathrm{O} 14^{\text {ix }}$ & 0.82 & 2.09 & $2.855(4)$ & 154 \\
$\mathrm{O} 21 W-\mathrm{H} 21 A \cdots \mathrm{O} 5^{\text {iv }}$ & 0.82 & 2.02 & $2.773(4)$ & 153 \\
$\mathrm{O} 21 W-\mathrm{H} 21 A \cdots \mathrm{O} 15$ & 0.82 & 2.50 & $2.914(4)$ & 112 \\
$\mathrm{O} 21 W-\mathrm{H} 21 B \cdots \mathrm{O} 12^{\text {iv }}$ & 0.82 & 1.93 & $2.751(4)$ & 174 \\
\hline
\end{tabular}

Symmetry codes: (iv) $x,-y-\frac{1}{2},+z-\frac{1}{2}$; (v) $x, y-1, z$; (vi) $x,-y-\frac{1}{2},+z+\frac{1}{2}$; (viii) $-x,-y,-z$; (ix) $-x+1,-y,-z-1$.

Water $\mathrm{H}$ atoms were positioned geometrically $(\mathrm{O}-\mathrm{H}=0.82 \AA)$ and refined as riding, with the constraint $U_{\text {iso }}(\mathrm{H})=1.5 U_{\text {eq }}$ (carrier) applied. The highest electron-density peak is located $0.79 \AA$ from atom S3.

Data collection: SMART (Bruker, 1998); cell refinement: SMART; data reduction: SAINT (Bruker, 1998); program(s) used to solve structure: SHELXS97 (Sheldrick, 1997); program(s) used to refine structure: SHELXL97 (Sheldrick, 1997); molecular graphics: ORTEP3 (Farrugia, 1997) and CAMERON (Watkin et al., 1993); software used to prepare material for publication: Win $G X$ (Farrugia, 1999) and PLATON (Spek, 2003).

The authors thank the Department of Science and Technology, India (IRHPA-DST), for providing the CCD facility at the Indian Institute of Science.

\section{References}

Bruker (1998). SMART (Version 5.0) and SAINT (Version 6.02). Bruker AXS Inc. Madison Wisconsin, USA.

Farrugia, L. J. (1997). J. Appl. Cryst. 30, 565.

Farrugia, L. J. (1999). J. Appl. Cryst. 32, 837-838.

Hidalgon, A., Veintemillas, S., Rodriguez-Clemente, R., Molins, E., Balarew, C., Keremidchieva, B. \& Spasov, V. (1996). Z. Kristallogr. 211, 153-157.

Sheldrick, G. M. (1996). SADABS. University of Göttingen, Germany.

Sheldrick, G. M. (1997). SHELXS and SHELXL97. University of Göttingen, Germany.

Spek, A. L. (2003). J. Appl. Cryst. 36, 7-13.

Watkin, D. M., Pearce, L. \& Prout, C. K. (1993). CAMERON. Chemical Crystallography Laboratory, University of Oxford, England. 